\title{
Centre-surround organization of fast sensorimotor integration in human motor hand area
}

Dubbioso, Raffaele; Raffin, Estelle; Karabanov, Anke; Thielscher, Axel; Siebner, Hartwig Roman

Published in:

Neurolmage

Link to article, DOI:

10.1016/j.neuroimage.2017.06.063

Publication date:

2017

Document Version

Peer reviewed version

Link back to DTU Orbit

Citation (APA):

Dubbioso, R., Raffin, E., Karabanov, A., Thielscher, A., \& Siebner, H. R. (2017). Centre-surround organization of fast sensorimotor integration in human motor hand area. Neurolmage, 158, 37-47.

https://doi.org/10.1016/j.neuroimage.2017.06.063

\section{General rights}

Copyright and moral rights for the publications made accessible in the public portal are retained by the authors and/or other copyright owners and it is a condition of accessing publications that users recognise and abide by the legal requirements associated with these rights.

- Users may download and print one copy of any publication from the public portal for the purpose of private study or research.

- You may not further distribute the material or use it for any profit-making activity or commercial gain

- You may freely distribute the URL identifying the publication in the public portal 


\section{Accepted Manuscript}

Centre-surround organization of fast sensorimotor integration in human motor hand area

Raffaele Dubbioso, Estelle Raffin, Anke Karabanov, Axel Thielscher, Hartwig Roman Siebner

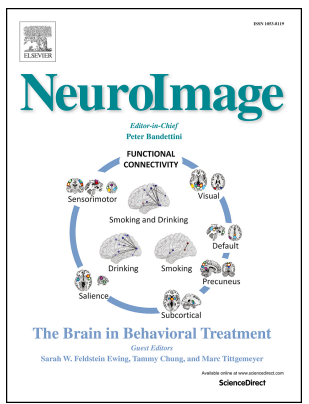

PII:

S1053-8119(17)30533-5

DOI:

10.1016/j.neuroimage.2017.06.063

Reference: $\quad$ YNIMG 14145

To appear in: Neurolmage

Received Date: 8 April 2017

Accepted Date: 22 June 2017

Please cite this article as: Dubbioso, R., Raffin, E., Karabanov, A., Thielscher, A., Siebner, H.R., Centresurround organization of fast sensorimotor integration in human motor hand area, Neurolmage (2017), doi: 10.1016/j.neuroimage.2017.06.063.

This is a PDF file of an unedited manuscript that has been accepted for publication. As a service to our customers we are providing this early version of the manuscript. The manuscript will undergo copyediting, typesetting, and review of the resulting proof before it is published in its final form. Please note that during the production process errors may be discovered which could affect the content, and all legal disclaimers that apply to the journal pertain. 


\section{Centre-surround organization of fast sensorimotor integration in human motor hand area}

Raffaele Dubbioso $^{1,2^{*}}$, Estelle Raffin ${ }^{1,3}$, Anke Karabanov ${ }^{1}$, Axel Thielscher ${ }^{1,4}$,

Hartwig Roman Siebner ${ }^{1,5}$.

${ }^{1}$ Danish Research Centre for Magnetic Resonance, Centre for Functional and Diagnostic Imaging and Research, Copenhagen University Hospital Hvidovre, Hvidovre, Denmark.

${ }^{2}$ Department of Neurosciences, Reproductive Sciences and Odontostomatology, University Federico II of Naples, Italy.

${ }^{3}$ INSERM, U1216, Grenoble Institut des Neurosciences, GIN, F-38000 Grenoble, France

${ }^{4}$ Department of Electrical Engineering, Technical University of Denmark, Kongens Lyngby, Denmark.

${ }^{5}$ Department of Neurology, Copenhagen University Hospital Bispebjerg, Copenhagen, Denmark.

Title characters count: 87; abstract word count: 200; paper word count: 7081; tables: 1; figures: 5 .

Conflict of Interest: Hartwig R. Siebner has received honoraria as editor from Elsevier Publishers, Amsterdam, The Netherlands and Springer Publishing, Stuttgart, Germany, and has received a research fund from Biogen-idec. The remaining authors have no conflicts of interest.

\section{*Corresponding author:}

Raffaele Dubbioso, MD,

Danish Research Center for Magnetic Resonance (DRCMR),

Center for Functional and Diagnostic Imaging and Research,

Copenhagen University Hospital Hvidovre

Kettegård Allé 30, DK-2650 Hvidovre, Denmark.

Phone: +45 3862 3862, e-mail: rafdubbioso@gmail.com 


\begin{abstract}
Using the short-latency afferent inhibition (SAI) paradigm, transcranial magnetic stimulation (TMS) of the primary motor hand area $\left(\mathrm{M} 1_{\mathrm{HAND}}\right)$ can probe how sensory input from limbs modulates corticomotor output in humans. Here we applied a novel TMS mapping approach to chart the spatial representation of SAI in human hand-knob. We hypothesized SAI is somatotopically expressed in $\mathrm{M} 1_{\mathrm{HAND}}$ depending on both the site of peripheral electrical nerve stimulation and the cortical spot targeted by TMS within M1 $1_{\mathrm{HAND}}$. The left index or little finger was stimulated $23 \mathrm{~ms}$ before focal single-pulse TMS of the right $\mathrm{M}_{\mathrm{HAND}}$. Using frameless stereotaxy, we applied biphasic-TMS pulses at seven stimulation positions above right $\mathrm{M} 1_{\mathrm{HAND}}$ and recorded the motor evoked potentials (MEPs) from relaxed left first-dorsal-interosseous (FDI) and abductor-digiti-minimi (ADM) muscles. Homotopic stimulation of the finger close to the muscle targeted by TMS revealed a somatotopic expression of afferent inhibition matching the somatotopic representation of unconditioned MEPs (homotopic SAI). Conversely, heterotopic stimulation of a finger distant to the muscle targeted by TMS induced short-latency afferent facilitation (SAF) of

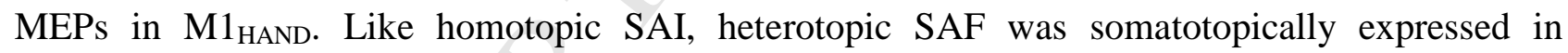
M1 $1_{\text {HAND. }}$ Together, the results provide first-time evidence that fast sensorimotor integration involves centre-inhibition and surround-facilitation in human $\mathrm{M} 1_{\mathrm{HAND}}$.
\end{abstract}

Keywords: surround facilitation, Short-latency afferent inhibition, somatotopy, cortical mapping, transcranial magnetic stimulation. 


\section{Abbreviations}

SAI: short-latency afferent inhibition

SAF: short-latency afferent facilitation

M1 HAND: primary motor hand area

FDI: first dorsal interosseous muscle

ADM: abductor digiti minimi muscle

PT: perceptual threshold

MCV: maximum voluntary contraction

AUC: area under the curve

WMP: weighted mean position 


\section{Introduction}

Humans have the capacity to acquire a large repertoire of fine motor skills, which require the flexible integration of sensory signals into motor commands. Such integration takes place in the primary sensorimotor cortex. The primary somatosensory cortex (S1) and primary motor cortex (M1) are commonly considered to be functionally segregated regions with S1 processing sensory input and M1 encoding motor output (Sanes and Donoghue, 2000). However, recent lines of research showed that both, M1 and S1 jointly contribute to sensory and motor aspects of motor control (Hatsopoulos and Suminski, 2011). In animals, invasive recordings of cortical activity showed that M1 directly receives somatosensory input enabling highly flexible, context-dependent encoding of movement kinematics (Balzamo et al., 2004; Churchland and Shenoy, 2007; Ferezou et al., 2007; Hatsopoulos et al., 2007). Conversely, S1 actively participates in motor control, for instance driving whisker retraction in mice (Matyas et al., 2010; Petersen, 2014).

Influential concepts of sensorimotor integration stress an active influence of cortical sensory input on motor output and vice versa. Somatosensory inputs inform both, reflexive and volitional actions (Friston and Kiebel, 2009; Friston et al., 2009; Hommel, 2009). This comprises bodily feedback generated by the movement itself and somatosensory input signalling the consequence of a movement, for instance the haptic experience when manipulating an object. Conversely, motor output impacts on perception. Reciprocal sensorimotor interactions in the sensory-motor hand area $\left(\mathrm{SM} 1_{\mathrm{HAND}}\right)$ support cooperative interactions between associated sensory and motor events (Desmurget and Sirigu, 2015). Yet reciprocal sensorimotor interactions might also have "antagonistic" properties, causing active inhibition of sensorimotor events that lack contingent association. Indeed, the human $\mathrm{M} 1_{\mathrm{HAND}}$ expresses an inhibitory activation pattern around the central core of excitatory activation (i.e. centre-surround structure) which counteracts non-contingent mappings between sensory and motor events (Beck and Hallett, 2011). Surround inhibition has also been demonstrated in the $\mathrm{S} 1$ with the reciprocal inhibition of the somatosensory evoked potential amplitude evoked by median and ulnar nerve stimulation (Tinazzi et al., 2000). Surround inhibition 
mechanism in S1, resulting in a centre-surround organization, is thought to help sharpen sensory perceptions (Beck and Hallett, 2011). While sensorimotor synergies are theoretically well motivated, in vivo studies of these synergies in action in human $\mathrm{SM} 1_{\mathrm{HAND}}$ remain scarce.

Intracortical sensorimotor interactions can be probed using the short-latency afferent inhibition (SAI) paradigm (Tokimura et al., 2000). The amplitude of a motor evoked potential (MEP), induced by transcranial magnetic simulation (TMS) of $\mathrm{M} 1_{\mathrm{HAND}}$, is reduced by a peripheral electrical stimulus applied to a peripheral nerve of the contralateral hand 20-28 ms before the TMS pulse. Pharmacological TMS studies have shown that SAI exerts its inhibitory effects on the corticospinal neurons through the GABA-ergic interneurons in M1 $1_{\text {HAND }}$ (V Di Lazzaro et al., 2005; Vincenzo Di Lazzaro et al., 2005; Di Lazzaro and Ziemann, 2013). SAI is also a marker of cortical cholinergic activity, because anticholinergic drugs reduces the magnitude of SAI (Di Lazzaro et al., 2000). Further, patients with Alzheimer's disease show an attenuation of SAI, and the cholinesterase inhibitor rivastigmine can restore SAI (Di Lazzaro et al., 2004).

We recently introduced a novel mapping approach of muscle-specific representations in the M1 $1_{\text {HAND }}$ which aligns the position and orientation of the TMS coil to the individual shape of the central sulcus with the help of stereotactic neuronavigation (Raffin et al., 2015). Our sulcus-shape based mapping approach takes into account the individual curvature of the precentral knob that forms the human hand area (Yousry et al., 1997). In this study, we employed sulcus-shape based cortical mapping to examine how the human $\mathrm{M} 1_{\mathrm{HAND}}$ integrates sensory and motor signals. Using cutaneous electrical nerve stimulation, previous SAI studies showed stronger SAI when the electrical conditioning stimulus was applied to a digit located near the target muscle (homotopic stimulation) compared to digit stimulation distant from the target muscle (heterotopic stimulation) (Classen et al., 2000; Vincenzo Di Lazzaro et al., 2005; Tamburin et al., 2001). Building on these work, we tested the hypotheses that fast sensorimotor integration at the cortical level is mediated by topographically specific interactions, showing a centre-surround organization. We predicted that cutaneous electrical digit stimulation concurrently triggers homotopic "centre" representation of 
SAI and heterotopic "surround" representation of short-latency afferent facilitation (SAF) in the human M1 $_{\text {HAND }}$. Critically, we expected that the concurrently expressed representations of SAI and SAF display distinct spatial profiles, which match the muscle-specific somatotopy in $\mathrm{M} 1_{\mathrm{HAND}}$ (Experiment 1). Based on previous SAI studies (Classen et al., 2000), we further anticipated that this centre-surround organization of sensorimotor integration in M1 HAND depends on the sensorimotor state. In that study, tonic contraction attenuated SAI, suggesting that the short-latency afferent modulation as probed by the SAI paradigm is most prominent when afferents are stimulated in a "resting" motor state. We therefore hypothesized that selective pre-activation of the homotopic muscle will abolish surround facilitation (SAF) in the relaxed heterotopic muscle. Likewise, we anticipated that selective pre-activation of the heterotopic muscle will abolish homotopic inhibition (SAI) in the relaxed homotopic muscle.

\section{Materials and methods}

\subsection{Participants}

Fourteen healthy volunteers (mean age: $27.8 \pm 1.7 \mathrm{SE}, 5$ women) participated in the first experiment. Ten subjects also participated in the second experiment (mean age: $28.7 \pm 2.0 \mathrm{SE}, 4$ women). All participants were right handed as assessed by the Edinburgh handedness inventory (Oldfield, 1971) and had no history of neurological or psychiatric disorders. All subjects were screened for contraindications to TMS (Rossi et al., 2009). They all gave written informed consent to the experimental procedures. The study complied with the Helsinki declaration on human experimentation and was approved by the Ethics Committee of the Capital Region of Denmark (H15000551). 


\subsection{Shape-based neuronavigated TMS of the primary motor cortex}

On the same day of the TMS experiment, participants underwent structural high-resolution magnetic resonance imaging (MRI) of the whole brain at 3-Tesla (Verio, Siemens, Erlangen, Germany) to reconstruct individual brain surface for mapping. Structural MRI employed a threedimensional, T1-weighted magnetization prepared rapid acquisition gradient echo (MPRAGE) sequence consisting of 192 sagittal slices with $1 \mathrm{~mm}^{3}$ isotropic voxel resolution $(\mathrm{TR} / \mathrm{TE}=$ 2300/2.98 ms, TI = $1100 \mathrm{~ms} ; 256 \times 256$ matrix, flip angle $\left.9^{\circ}\right)$.

For shape-based TMS mapping, participants were seated comfortably in a chair and the TMS coil position was continuously monitored by a frameless neuronavigation system (Localite, Sankt Augustin, Germany). The brain surface was automatically reconstructed from the T1-weighted images using neuronavigation software (Localite, Sankt Augustin, Germany). The root mean square of difference between the co-registered anatomical landmarks estimated by the neuronavigation software was set below $2 \mathrm{~mm}$ for each subject to maintain positioning accuracy all along the experiment.

TMS target locations in the precentral gyrus were marked prior to the experiment on the segmented brain of each subject. The right $\mathrm{M} 1_{\mathrm{HAND}}$ was identified by a trained investigator $(\mathrm{RD})$ using the characteristic knob-like shape of the sulcus ("hand knob") as anatomic landmark (Yousry et al., 1997). The investigator placed seven targets in the posterior part of the crown of the precentral

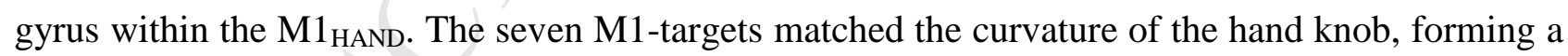
line of equidistant targets every $10 \mathrm{~mm}$. Target 4 corresponded to the centre of the "hand knob" (Fig. 1A). For each target, coil orientation was adjusted to produce a current direction perpendicular to the central sulcus. The individual coil positioning parameters were stored in the neuronavigation software. Table 1 reports the MNI normalized mean coordinates provided by the neuronavigation system. 


\subsection{Surface electromyography (EMG)}

We recorded the electrical muscle activity of the left first dorsal interosseus (FDI) and abductor digiti minimi (ADM) muscle with surface electrodes (Ambu Neuroline 700, Ballerup, Denmark) arranged in a bipolar belly-tendon montage. The signals from the EMG electrodes were amplified, bandpass filtered $(5-3000 \mathrm{~Hz})$, digitized at a frequency of $5 \mathrm{kHz}$, and stored in a laboratory computer for later offline analysis, using Signal software and CED 1401 hardware (Cambridge Electronic Design, Cambridge, UK).

\subsection{Peripheral electrical digit stimulation}

Peripheral electrical stimuli were given to the fingers through bipolar ring electrodes strapped around the left index and little fingers. We applied square pulses of $200 \mu$ s duration with the cathode positioned at the proximal and the anode positioned at the distal interphalangeal joint (Digitimer stimulator, Model DS7A, Hertfordshire, England). The peripheral stimulation was applied $23 \mathrm{~ms}$ prior to the TMS pulse to elicit SAI (Tokimura et al., 2000). In each participant, perceptual threshold (PT) was determined for the little and index fingers by delivering a series of stimuli at increasing intensity from $2 \mathrm{~mA}$ in steps of $1 \mathrm{~mA}$. The PT was defined as the minimal intensity of stimulation perceived by the participant in 10 of 10 consecutive stimuli (Dubbioso et al., 2015; Manganelli et al., 2013). Stimulation intensity was set to $100 \%, 200 \%$ or $300 \%$ of individual PT (PT100\%, PT200\%, PT300\%) of each finger for electrical digit stimulation in the first experiment (rest condition) and to PT300\% in the second experiment (contraction condition). We didn't used higher stimulus intensities to preserve a topographically confined conditioning effect in M1 $1_{\text {HAND }}$ (Tamburin, Manganotti, Zanette, \& Fiaschi, 2001). None of the subjects perceived the peripheral nerve stimulation as painful.

\subsection{Transcranial magnetic stimulation}


Single-pulse TMS was performed using a MagPro X100 stimulator (Magventure, Skovlunde, Denmark) connected to a cooled-MC-B35 figure-of-eight coil with windings of $35 \mathrm{~mm}$ diameter. In order to keep TMS as spatially confined as possible, we used a biphasic pulse configuration generating an antero-posterior followed by postero-anterior (AP-PA) current in the brain, because this pulse configuration allowed effective suprathreshold stimulation of $\mathrm{M} 1_{\text {HAND }}$ at the lowest possible stimulation intensity (Lang et al., 2006). We first located the target position among our seven predefined locations where TMS elicited the maximal MEP in the left ADM muscle (i.e. the ADM hotspot). We then placed the coil on the ADM hotspot with a 90-degree angle relative to the individual central sulcus shape (Fig. 1A) and determined the intensity of TMS stimulator in order to get an average MEP amplitude in the ADM muscle around $0.2-0.5 \mathrm{mV}$ by using the MaximumLikelihood Strategy Parameter Estimation by Sequential Testing (MLS-PEST) approach (Awiszus, 2003). TMS was applied at this individually adjusted stimulus intensity in Experiments 1 and 2. It had been previously shown that a test MEP amplitude varying between 0.2 and $1 \mathrm{mV}$ did not influence the relative magnitude of SAI (Udupa et al., 2014, 2009).

\subsection{Mapping procedures}

Experiments 1 and 2 were carried out on separate days at least one week apart. For each of the seven targets, we applied 20 pulses delivered at inter-stimulus intervals jittered between 4 and $5 \mathrm{~s}$. In each stimulation block, the order of conditioned (10 pulses preceded by electrical stimulation) and unconditioned MEPs (Test stimulus alone, 10 pulses) was pseudo-randomized. Within each session, the order of stimulated targets was pseudo-randomized and counterbalanced across subjects.

\subsubsection{Experiment 1}


The experiment was designed to evaluate the effect of different conditioning peripheral stimulation intensities $(100 \%, 200 \%$ and $300 \%$ of the individual PT) on the somatotopic representation of SAI during heterotopic and homotopic stimulation. For homotopic stimulation, we applied the conditioning peripheral stimulus to the digit close to the muscle targeted by TMS. Conversely, for heterotopic stimulation we delivered the conditioning stimulus to the digit distant from the muscle targeted by TMS. For example, electrical digit stimulation of the index finger was homotopic with respect to the FDI muscle and heterotopic with respect to the ADM muscle (Fig. 1B). Likewise, electrical digit stimulation of the little finger was homotopic with respect to the ADM muscle and heterotopic with respect to the FDI muscle (Fig. 1B).

\subsubsection{Experiment 2}

Experiment 2 employed sulcus-shape informed SAI mapping during tonic contraction of FDI and ADM muscles to test how motor activity of one intrinsic hand muscle impacts on the SAI profile of the "surrounding" hand muscle staying relaxed. We performed SAI mapping during two active conditions: (1) isometric contraction of the FDI muscle with the "surrounding" ADM muscle being relaxed, (2) isometric contraction of the ADM muscle with the "surrounding" FDI muscle being relaxed (Fig. 1B).

Participants maximally abducted their fingers against a force-sensor device and we calculated 10\% of their maximum voluntary contraction (MVC) for the index and little finger separately. We trained participants to produce a constant isometric contraction at around $10 \%$ of MVC based on continuous visual feedback displayed on an oscilloscope, while keeping the surrounding muscle completely relaxed. Participants continuously received feedback of the EMG activity of the FDI and ADM muscles. We discarded trials in which background EMG activity in the "surrounding" muscle exceeded $0.1 \mathrm{mV}$. To avoid fatigue, each sequence of isometric contractions lasted $45 \mathrm{~s}$ followed by a 30-second break. We allowed additional resting periods, if needed by the subject. Pre-activation conditions with the index or little finger were counterbalanced across subjects. 


\section{Data analyses}

MEP trials were visually inspected and trials with visible voluntary motor activity were removed in recordings performed at rest. For each trial, peak-to-peak MEP amplitude of each MEP was determined in the time window between 10 and $30 \mathrm{~ms}$ after the TMS stimulus (Signal software, version 6.04 for Windows, Cambridge Electronic Design, Cambridge, UK). We generated muscle excitability profiles for the test stimulus alone (unconditioned MEP) and the MEP preceded by peripheral stimulation along the seven targets (conditioned MEP) under the various conditions.

Using the conditioned or unconditioned mean MEP amplitude for each subject and stimulation site as the dependent variable, we computed a repeated measure ANOVA to model the distribution of the conditioned and unconditioned MEPs amplitudes recorded from the ADM and FDI muscles across targets and to test for differences between conditions at the group level using a repeated measure ANOVA. We computed separate ANOVAs for each experiment. In experiment 1, the ANOVA included the within-subject factors cortical target (target site 1 - 7), muscle (ADM vs FDI muscle), intensity of peripheral electrical stimulation $(100 \%, 200 \%$, or $300 \%$ of individual PT), and site of peripheral stimulation (index versus little finger).

In experiment 2, the ANOVA included the within-subject factors cortical target (target site 1 - 7), muscle (ADM vs FDI muscle), site of peripheral stimulation (index versus little finger), and state (tonic contraction versus relaxation).

We computed two additional indicators of cortical excitability. First, we calculated the area under the curve (AUC) to assess the effect of different peripheral stimulation intensities during heterotopic and homotopic stimulation. AUC was calculated according to the following formula:

$$
A U C=\sum_{k=1}^{7} \text { Mean MEP Amplitude Target }(\mathrm{k})
$$


Target (k) refers to each target's number (from 1 to 7) and Mean MEP Amplitude Target (k) refers to the mean peak-to-peak Motor-Evoked-Potential amplitudes at each target (from target 1 to target 7). This indicator reflects the up or down regulation of the global corticospinal excitability recorded from a single muscle (Raffin et al., 2015). A ratio between the AUC of the conditioned muscle profiles $\left(\mathrm{AUC}_{\mathrm{c}}\right)$ and the $\mathrm{AUC}$ of the unconditioned muscle profiles $\left(\mathrm{AUC}_{\mathrm{u}}\right)$ higher than 1 indicated a facilitatory effect of the peripheral stimulation on the MEP amplitudes whereas a value lower than 1 showed inhibition.

Second, we computed the amplitude-weighted mean position, denoted here as weighted mean position (WMP) of each muscle profile to return the one-dimensional muscle location along M1 $1_{\text {HAND }}$ (Raffin et al., 2015). WMP was calculated according to the following formula:

$$
W M P=\frac{\sum_{k=1}^{7} \text { Target }(k) * \text { Mean MEP Amplitude Target }(k)}{\sum_{k=1}^{7} \text { Mean MEP Amplitude Target }(\mathbf{k})}
$$

Distinct weighted mean positions associated with the two muscle profiles suggest distinct corticomotor representations for the two muscles. Along the same line of reasoning, differences in WMP across conditions for a single muscle indicate context-dependent shifts of the muscle excitability profile. The $\mathrm{AUC}_{\mathrm{c}} / \mathrm{AUC}_{\mathrm{u}}$ ratio and the Weighted Mean Positions of each muscle were entered into two separate ANOVAs with different within-subject factors according to the aim of the analysis (see Results part).

Finally, we performed a correlation analysis between the peak value of the $\mathrm{AUC}_{\mathrm{C}} / \mathrm{AUC}_{\mathrm{U}}$ ratio of the homotopic (inhibitory) and heterotopic (facilitatory) stimulation centred on targets 3,4 and 5 of the two muscles using Pearson correlation coefficient. We chose these three targets of the sulcusshape based mapping line, since these targets were located exactly in the medial, central, and lateral part of the cortical hand knob, respectively.

All statistical analyses used IBM SPSS Statistics software (Version 22 for Windows, New York City, USA). Normal distribution of all variables was verified by means of a Kolmogorov and Smirnov test. Alpha inflation due to multiple comparisons was controlled using Bonferroni 
correction when appropriate. We used the Mauchly's Test to test for sphericity and the GreenhouseGeisser correction method to correct for non-sphericity. Group data are given as mean \pm standard error of the mean (SEM).

\section{Results}

\subsection{Experiment 1}

Experiment 1 addressed the somatotopy of short-latency sensorimotor integration in $\mathrm{M}_{\mathrm{HAND}}$ during heterotopic and homotopic stimulation (Fig. 2A).

\subsubsection{Somatotopic representation of the unconditioned MEPS}

We first performed a repeated measure ANOVA which used the unconditioned MEP amplitude as independent variable to confirm the somatotopic representation of the FDI and ADM muscle (Raffin et al., 2015) and to assess the contextual impact of the intensity and site of peripheral stimulation in conditioned trials on these representations. We treated cortical target, muscle, intensity, and site of peripheral stimulation as within-subject factors. In agreement with previous work (Raffin et al., 2015), we found a somatotopic gradient of muscle arrangement along the hand motor area, with the FDI muscle being represented more laterally than the ADM muscle (Fig. 2B). Accordingly, the ANOVA yielded a main effect of muscle $\left(\mathrm{F}_{(1,13)}=23.256, \mathrm{p}<0.001\right)$, target $\left(\mathrm{F}_{(2.62,34.1)}=15.643, \mathrm{p}<0.001\right.$; Mauchly's Test of Sphericity: $\chi_{(20)}^{2}=87.1, \mathrm{p}=<0.001$; GreenhouseGeisser correction: $\varepsilon=0.437)$ and a significant interaction between muscle and target $\left(\mathrm{F}_{(2.1,27.3)}=\right.$ 9.094, $\mathrm{p}=0.001$; Mauchly's Test of Sphericity: $\chi_{(20)}^{2}=123.05, \mathrm{p}=<0.001$; Greenhouse-Geisser correction: $\varepsilon=0.350$ ). The spatial dissociation of the two muscle profiles was further corroborated by a significant main effect of muscle in the ANOVA comparing the weighted mean positions of each of the two curves muscle $\left(\mathrm{F}_{(1,13)}=42.873, \mathrm{p}<0.001\right)$. Importantly, we found no significant impact of the intensity and site of peripheral stimulation on the somatotopy, represented by the weighted mean positions values, of the unconditioned muscle representations (i.e., in the absence of 
peripheral stimulation). Indeed, using the mean MEP amplitude as dependent variable, ANOVA showed neither an interaction among target, intensity of peripheral stimulation, and muscle nor among target, site of peripheral stimulation, and muscle ( $\mathrm{p}>0.6)$. There was also no interaction between intensity of peripheral stimulation and muscle or site of peripheral stimulation and muscle in the ANOVA testing condition-specific effects on weighted mean positions $(\mathrm{p}>0.5)$. Together, these results replicate the well-known somatotopic representation of the FDI and ADM muscle in the $\mathrm{M} 1_{\mathrm{HAND}}$. The results also exclude a context effect of the SAI paradigm on the ability to map the somatotopy of corticomotor representations with the unconditioned test pulses. In other words, the normal somatotopy of corticomotor representations in $\mathrm{M}_{\mathrm{HAND}}$ can still be mapped with unconditioned single-pulse TMS, even when these unconditioned TMS pulses are intermingled with conditioned TMS pulses.

\subsubsection{Somatotopic representations of short-latency afferent inhibition and facilitation}

The main ANOVA focused on the conditioned MEPs to test for a somatotopic representation of SAI treating target, muscle, site and intensity of peripheral stimulation as within-subject factors. We found a distinct medio-lateral distribution of the conditioned MEPs amplitudes for the FDI and $\mathrm{ADM}$ muscles as demonstrated by a significant interaction between target and muscle $\left(\mathrm{F}_{(6,78)}=\right.$ 8.843, $\mathrm{p}<0.001)$. This muscle-specific somatotopic representation was influenced by the site and intensity of peripheral electrical stimulation as reflected by a significant interaction among target, site and intensity of peripheral stimulation, and muscle $\left(\mathrm{F}_{(4.48,58.26)}=3.589, \mathrm{p}=0.009 ;\right.$ Mauchly's Test of Sphericity: $\chi_{(20)}^{2}=87.1, p=<0.001$; Greenhouse-Geisser correction: $\left.\varepsilon=0.437\right)$.

To quantify the effect of different PT intensities during heterotopic and homotopic stimulation on the SAI profiles, we computed the AUC for each of the conditioned MEP profiles (see data analysis). We then determined the ratio between the AUC of the conditioned MEP amplitudes $\left(\mathrm{AUC}_{\mathrm{c}}\right)$ and the AUC of the unconditioned MEP amplitudes $\left(\mathrm{AUC}_{\mathrm{u}}\right)$ and finally computed a repeated measure ANOVA with $\mathrm{AUC}_{\mathrm{c}} / \mathrm{AUC}_{\mathrm{u}}$ as dependent variable and muscle, site and intensity 
of peripheral stimulation as within-subject factors. The analysis revealed an intensity-dependent effect of the type of stimulation (i.e. homotopic or heterotopic stimulation) on the SAI profiles recorded from the two muscles as reflected by an interaction between site and intensity of peripheral stimulation and muscle $\left(\mathrm{F}_{(2,26)}=17.812, \mathrm{p}<0.001\right)$.

For homotopic stimulation, a higher intensity of peripheral stimulation caused greater inhibition of the conditioned MEP amplitude. Bonferroni-corrected paired comparisons showed that homotopic stimulation induced a reduction of the SAI ratio at $300 \%$ of PT for both muscles compared to the two other intensities, indicating a dose-dependent inhibitory effect (Fig. 2C). There was a consistent SAI at an intensity of $300 \%$, but not at $100 \%$ PT. The inhibitory conditioning effect at $300 \%$ PT was significantly greater than SAI at $100 \%$ PT for the FDI muscle (PT300\%: mean $=0.72 \pm 0.05$; PT100\%: mean=1.04 \pm 0.06; $\mathrm{p}=0.001)$, and ADM muscle (PT300\%: $0.86 \pm 0.04 ;$ PT200\%: $1.07 \pm$ $0.05 ; p=0.041)$

In contrast to homotopic stimulation, heterotopic stimulation had a facilitatory effect on $\mathrm{AUC}_{\mathrm{c}} / \mathrm{AUC}_{\mathrm{u}}$ ratio (Fig. 2D). In analogy to $\mathrm{SAI}$, we refer to this conditioning effect as short-latency afferent facilitation (SAF). Like SAI, SAF depended on the intensity of peripheral stimulation (Fig. 2D). For both muscles, post-hoc testing revealed a significant increment of the $\mathrm{AUC}_{\mathrm{c}} / \mathrm{AUC} \mathrm{C}_{\mathrm{u}}$ ratio at an intensity of 300\% PT compared to 200\% PT (FDI: PT300\%: $1.15 \pm 0.06$; PT200\%: $0.89 \pm 0.06$; $\mathrm{p}=0.026 ;$ ADM: РT300\%: $1.09 \pm 0.05 ;$ PT200\%: $0.94 \pm 0.04 ; \mathrm{p}=0.019)$.

We measured the spatial dissociation of the two SAI profiles using the weighted mean positions to examine the muscle-specificity of sensorimotor integration. Since the largest modulatory effect on the conditioned MEP amplitude was present at a stimulus intensity of $300 \%$ PT, we only considered the homotopic SAI and heterotopic SAF profiles evoked with an electrical digital stimulus that matched $300 \%$ of individual PT. We computed a repeated measure ANOVA on the weighted mean position with site of peripheral stimulation and muscle as within-subject factors. We found a mediolateral dissociation of the sensorimotor integration associated with the FDI and ADM muscle as evidenced by a significant main effect of "Muscle" $\left(\mathrm{F}_{(1,13)}=25.209, \mathrm{p}<0.001\right)$ with the FDI 
muscle being located more laterally and the ADM muscle more medially along the central sulcus (Fig. 3A-B). Importantly we did not find any main effect of site of peripheral stimulation $\left(\mathrm{F}_{(1,13)}=\right.$ $0.521, \mathrm{p}=0.483)$ or a site of peripheral stimulation by muscle interaction $\left(\mathrm{F}_{(1,13)}=0.366, \mathrm{p}=\right.$ 0.555), suggesting that the somatotopic arrangement were similar for the homotopic or heterotopic stimulation (Fig. 3C-D).

\subsection{Experiment 2}

Experiment 2 was designed to assess how tonic muscle contraction of one of the two target muscles impacts on the sensorimotor interactions in the "surrounding" relaxed muscle revealed by heterotopic and homotopic stimulation.

\subsubsection{Somatotopic representation of the unconditioned MEPS}

We first tested whether the site of peripheral finger stimulation (stimulation of index versus little finger) or the motor context (rest versus tonic contraction of the non-target muscle) influenced the somatotopy of corticomotor representations, as revealed by the cortical test pulse alone without afferent conditioning stimulation. To this end, we computed a repeated measure ANOVA with the unconditioned MEP amplitude as dependent variable and target, muscle, site of peripheral stimulation and state as within-subject factors. Like in experiment 1 , there was a main effect of muscle $\left(\mathrm{F}_{(1,8)}=21.343, \mathrm{p}=0.002\right)$, target $\left(\mathrm{F}_{(6,48)}=14.747, \mathrm{p}=0.001\right)$ as well as an interaction between muscle and target $\left(\mathrm{F}_{(1.8,14.41)}=7.779, \mathrm{p}=0.006\right.$; Mauchly's Test of Sphericity: $\chi_{(20)}^{2}=$ 74.301, $\mathrm{p}=<0.001$; Greenhouse-Geisser correction: $\varepsilon=0.300$ ). The ANOVA revealed no higherorder interactions between muscle and target, involving additional factors (i.e., site of peripheral stimulation or state).

\subsubsection{Effect of tonic muscle contraction on heterotopic SAF and homotopic SAI}


We computed a repeated measure ANOVA to examine whether facilitation (SAF) or inhibition (SAI) that is present at rest is also present during tonic contraction of a different hand muscle. For the three cortical sites corresponding to the hand knob (i.e., target positions 3, 4, and 5), we pooled the $\mathrm{AUC}_{\mathrm{c}} / \mathrm{AUC}_{\mathrm{u}}$ ratios of the relaxed surrounding muscle together and used this value as dependent variable. The factors muscle, site of peripheral stimulation and state were within-subject factors. We found that selective tonic contraction of an intrinsic hand muscle abolished both, the facilitatory effect of heterotopic stimulation and the inhibitory effect of homotopic stimulation in the surrounding relaxed muscles (Fig. 4A). This effect resulted in a significant interaction between muscle and site of stimulation $\left(\mathrm{F}_{(1,9)}=62.038, \mathrm{p}<0.001\right)$ and among muscle, site of peripheral stimulation and state $\left(\mathrm{F}_{(1,9)}=72.471, \mathrm{p}<0.001\right)$. The Bonferroni-corrected paired comparisons for each muscle confirmed that tonic contraction induced a significant decrease in SAF evoked by heterotopic stimulation (FDI muscle: paired t-test: $\mathrm{t}_{(9)}=-4.963, \mathrm{p}<0.001$; ADM muscle: paired $\mathrm{t}$ test: $\mathrm{t}_{(9)}=-2.780, \mathrm{p}=0.021$ ). The same was the case for SAI evoked by homotopic stimulation (FDI muscle: paired t-test: $\mathrm{t}_{(9)}=6.291, \mathrm{p}<0.001$; ADM muscle : paired t-test: $\mathrm{t}_{(9)}=3.612, \mathrm{p}=0.006$; Fig. 4B).

We tested whether contraction-induced reduction of SAF in the relaxed heterotopic muscle and SAI in the relaxed homotopic muscle had a specific spatial distribution. The conditioned MEP amplitudes, recorded from the relaxed muscle, entered in a repeated measure ANOVA with target, muscle, and site of peripheral stimulation as within-subject factors. We found a main effect of target $\left(\mathrm{F}_{(6,48)}=20.161, \mathrm{p}<0.001\right)$, muscle $\left(\mathrm{F}_{(1,8)}=6.908, \mathrm{p}=0.03\right)$ and a interaction between target and muscle $\left(\mathrm{F}_{(6,48)}=3.614, \mathrm{p}=0.046\right)$. These results show that the contraction induced decrease in SAF and SAI showed a spatial pattern that is specific to the cortical muscle representation (Fig. 4C). The spatial specificity of reduced SAF and SAI was also evident for the weighted mean positions of the cortical representations of the FDI and ADM muscles (Fig. 4D). There was a spatial dissociation between the two muscles for both, heterotopic stimulation (paired t-test: $\mathrm{t}_{(9)}=-3.372, \mathrm{p}$ $=0.008$ ) and homotopic stimulation (paired t-test: $\mathrm{t}_{(9)}=2.298, \mathrm{p}=0.047$ ). 


\subsection{Relationship between short-latency facilitation and inhibition}

We explored whether the magnitude of SAF, induced by heterotopic stimulation, scaled with the magnitude of SAI, induced by homotopic stimulation, within and between the two muscles. We identified the peak change in $\mathrm{AUC}_{\mathrm{c}} / \mathrm{AUC}_{\mathrm{u}}$ ratio evoked at an intensity of $300 \% \mathrm{PT}$ in the hand knob targets (i.e., target positions 3, 4 and 5) for heterotopic (facilitatory) and homotopic (inhibitory) stimulation.

The highest $\mathrm{AUC}_{\mathrm{c}} / \mathrm{AUC}_{\mathrm{u}}$ ratio evoked by heterotopic stimulation reflected maximal $\mathrm{SAF}$, whereas the lowest $\mathrm{AUC}_{\mathrm{c}} / \mathrm{AUC}_{\mathrm{u}}$ ratio evoked by homotopic stimulation indicated maximal SAI. When the little finger was stimulated, the magnitude of homotopic SAI scaled positively with heterotopic SAF (Fig. 5A). The stronger homotopic SAI in the ADM muscle, the stronger was heterotopic SAF in the FDI muscle, resulting in a significant negative correlation between maximal $\mathrm{AUC}_{\mathrm{c}} / \mathrm{AUC}_{\mathrm{u}}$ ratios elicited by heterotopic stimulation in the FDI muscle and by homotopic stimulation in the ADM muscle $\left(r=-0.791, p_{\text {corrected }}=0.005\right)$. This was not the case for stimulation of the index finger, where the magnitude of homotopic SAI in the FDI muscle showed no linear relationship with the magnitude of heterotopic SAF in the ADM muscle $\left(r=-0.104, p_{\text {uncorrected }}=0.724\right)$.

We also found that the maximal amount of SAI evoked in the intrinsic hand muscle correlated with each other (Fig. 5B). There was a positive linear relationship between maximal SAI evoked by homotopic stimulation in the FDI muscle and homotopic stimulation in the ADM muscle $(r=0.803$, $\left.\mathrm{p}_{\text {corrected }}=0.003\right)$. We found no other significant correlations between individual magnitudes of maximal SAI and SAF. Especially maximal SAF in one hand muscle did not predict maximal SAF in the other hand muscle. Further, the individual magnitude of SAF and SAI in the same hand muscle did not show a significant linear relation. The correlations between SAI and SAF were not driven by inter-individual differences in intensity of peripheral stimulation. Inter-individual variations were very small across subjects (mean $\pm \mathrm{SD}: 2.71 \pm 0.47 \mathrm{~mA}$ ). Further, there was no 
correlation between individual PT and the strength of inhibition in ADM and FDI muscles or between individual PT and the strength of facilitation in the FDI muscle ( $\left.\mathrm{p}_{\text {uncorrected }}>0.36\right)$.

\section{Discussion}

Combining a well-known conditioning-test TMS paradigm (Tokimura et al., 2000) with a novel sulcus-shape based TMS mapping approach (Raffin et al., 2015), we charted the cortical somatotopy of fast sensorimotor integration in human $M 1_{\mathrm{HAND}}$. Sulcus-shape based mapping revealed a centre-surround organization of short-latency sensorimotor integration in human M1 HAND. During full muscle relaxation, electrical digit stimulation concurrently activated a homotopic "centre" representation of SAI and a heterotopic "surround" representation of SAF. The concurrently expressed representations of SAI and SAF displayed distinct spatial profiles, which matched the muscle-specific somatotopy in $\mathrm{M} 1_{\mathrm{HAND}}$. Centre SAI and surround SAF vanished, when the "homotopic" muscle was tonically contracted. These results provide novel insights into the somatotopical arrangement of short-latency sensorimotor integration in human $\mathrm{M} 1_{\mathrm{HAND}}$

\subsection{Somatotopy of fast sensorimotor integration in the human M1 $1_{\text {HAND }}$}

Previous TMS studies, that investigated SAI with single-site TMS, consistently showed that the magnitude of short-latency MEP inhibition evoked by cutaneous electrical finger stimulation depended on the spatial relationship between the stimulated digit and the intrinsic hand muscle targeted with single-site TMS. A seminal electrophysiological study on sensorimotor integration in M1 $1_{\text {HAND }}$ found that SAI at ISIs of $25-30 \mathrm{~ms}$ was less pronounced, when the stimulated digit was distant to the intrinsic hand muscle targeted by TMS (Classen et al., 2000). The observation that homotopic digit stimulation results in stronger short-latency inhibition that heterotopic stimulation was replicated by two independent studies (Vincenzo Di Lazzaro et al., 2005; Tamburin et al., 2001). 
The present study significantly extends these single-site TMS studies into two directions. Firstly, sulcus-shape based mapping of $\mathrm{M}_{\mathrm{HAND}}$ revealed consistent MEP facilitation in the heterotopic muscle. Single-site TMS studies only noted an attenuation of SAI in the heterotopic muscle, but no consistent heterotopic facilitation. Here we used sulcus-shape based TMS mapping to show that the short-latency effects of electrical digit stimulation are indeed opposite in sign, producing shortlatency inhibitory effects in the homotopic muscle (SAI) and short-latency facilitatory effects in the heterotopic muscle (SAF). In contrast to previous single-site TMS studies on SAI, our experimental set-up allowed homotopic and heterotopic stimulation not only of the sensory input to but also of the motor output from the cortical hand area. Homotopic stimulation (e.g. sensory input from index finger and motor output to FDI muscle or sensory input from little finger and motor output to ADM muscle) probed the direct connection of homotopic sensorimotor cortical regions. Heterotopic stimulation (e.g. sensory input from little finger and motor output to FDI muscle or sensory input from index finger and motor output to $\mathrm{ADM}$ muscle) probed sensorimotor interaction between neighboring sensorimotor representations. These short-latency effects displayed a centre-surround pattern. Homotopic stimulation caused inhibition and heterotopic stimulation produced facilitation of corticomotor output in $\mathrm{M} 1_{\mathrm{HAND}}$ to the relaxed target muscle. Of note, single-digit stimulation of the little or index finger elicited opposite short-latency effects on mean MEP amplitude in the ADM and FDI muscle, respectively. Together, the results show that homotopic or heterotopic electrical stimulation rapidly shapes the excitability of fast-conducting corticospinal output inducing centre inhibition and surround facilitation, presumably via local intracortical circuits.

The second novel finding is that the regional magnitude of SAI and SAF show a medio-lateral gradient in the motor representation of $\mathrm{M} 1_{\mathrm{HAND}}$ which is analogous to the somatotopic profile of each muscle revealed by sulcus-shape based mapping with unconditioned single-pulse TMS. Critically, SAI was most prominent in the part of the M1 $1_{\mathrm{HAND}}$, where the homotopic muscle is most strongly represented. Likewise, SAF was most prominent in the part of the M1 $1_{\text {HAND }}$, where the heterotopic muscle is represented most strongly. In other words, SAI was maximal at the cortical 
"hot spot" of the homotopic muscle, while SAF was maximal at the cortical "hot spot" of the heterotopic muscle. The representational profiles of SAI and SAF support the notion, that fingerspecific sensory input unfolds its fast modulatory effects on muscle-specific motor output in human $\mathrm{M} 1_{\text {HAND }}$ within the spatial frame of the existing cortical motor representations.

Electrical stimulation of finger II and finger V produced opposite effects on SAI or SAF and this pattern was found for the FDI and ADM representation in $\mathrm{M}_{\mathrm{HAND}}$. It should be noted that the intensity of the TMS pulse was identical for all cortical stimulation sites. We did not adjust the stimulus intensity of the test pulse to evoke non-conditioned test MEPs of equal mean amplitude across test sites because this would have blurred the spatial resolution of TMS mapping. Our procedure might raise the concern that the somatotopic effects of peripheral conditioning on shortlatency sensorimotor modulation of MEP amplitudes might be an epiphenomenon of the somatotopy of the corticomotor muscle representations as revealed by single-pulse TMS (Raffin et al., 2015). We argue that the somatotopy of SAI and SAF can't be attributed to size variations of the test response at a given cortical site. Critically, the somatotopic effect emerged as a bi-directional modulation of the unconditioned test MEP elicited at the spatial peak of the respective musclespecific corticomotor representation. Hence, the somatotopic effect of peripheral stimulation was not confounded by any differences in amplitude of the test response, but reveals a somatotopically specific integration of afferent input for both corticomotor muscle representations (FDI and ADM) in human $\mathrm{M} 1_{\mathrm{HAND}}$.

The finding that SAF was less pronounced at the cortical "hot spot" of the homotopic muscle may account for the fact that previous single-site TMS studies failed to detect consistent SAF over the hot spot of the homotopic muscle at the group level (Classen et al., 2000; Vincenzo Di Lazzaro et al., 2005; Tamburin et al., 2001).

Using diffusion MRI, a tractography study demonstrated that the motor and somatosensory homunculi are directly connected through short U-shaped fibres running beneath the central sulcus in humans (Catani et al., 2012). The pattern of distribution of these fibres follows the topographical 
organization of M1 and S1 with a larger amount of connections between homotopic body parts. This pattern found in humans matches findings obtained in animals, showing that the projections from S1 are topographically organized and terminate mainly in homologous M1 body part representations (Fabri and Burton, 1991; Izraeli and Porter, 1995). However, these studies also showed that focal sites in the M1 receive projections from S1 that represent neighboring body parts (Fabri and Burton, 1991; Izraeli and Porter, 1995). Our functional mapping results are in good agreement with the somatotopic arrangement of anatomical connections between S1 and M1 and have implications for the functional integration of sensory input and motor output in manual motor control. The spatial pattern of sensory-to-motor interactions indicate a capability of human $\mathrm{M} 1_{\mathrm{HAND}}$ to implement focal sensory input from the digits to concurrently tune the output from multiple (homotopic and heterotopic) motor representations. As discussed above, this type of sensorimotor integration may involve the $\mathrm{S} 1_{\mathrm{HAND}}$ and cortico-cortical connections between $\mathrm{S} 1$ and $\mathrm{M} 1$ and then reaches the motor cortex. Alternatively, these types of rapid sensory-to-motor interactions may primarily involve intracortical processing in $\mathrm{M} 1_{\mathrm{HAND}}$. The latter possibility is neuroanatomically possible, as there is cumulating evidence that the M1 receives direct sensory input through the thalamus bypassing the sensory cortex (Barbas and Garcìa-Cabezas, 2015; Bopp et al., 2017; Yamawaki et al., 2014)

The topographic distribution for homotopic and heterotopic stimulation depended on the intensity of afferent stimulation. Homotopic SAI and heterotopic SAF gradually emerged when increasing the intensity of electrical digit stimulation from $100 \%$ to $300 \%$ of individual perceptual threshold. This finding is in accordance with a previous single-site TMS study of SAI (Tamburin et al., 2001). The lack of SAI and SAF at lower intensities is most likely due to a failure to activate a sufficient number of cutaneous nerve fibers to produce a consistent modulation of corticomotor output in M1 HAND. Alternatively, sensorimotor integration patterns might fundamentally differ for close to threshold afferent input or input that is clearly suprathreshold but does not induce pain sensations. It is conceivable that the fast center-surround, inhibition-facilitation pattern might reflect a 
sensorimotor response pattern to a potentially harmful, externally induced sensory input to a finger. Such a rapid sensorimotor response pattern of local motor inhibition and neighboring motor facilitation is more relevant the higher the intensity of the focal sensory input. In this context, we wish to emphasize that the electrical stimuli did not induce pain. Accordingly, the short-latency of the SAI and SAF effects points to rapid sensorimotor integration mediated by fast conducting afferent sensory fibers.

At the individual level, the magnitude of homotopic SAI in the FDI and ADM muscle showed a strong linear relationship. This suggests that the excitability of GABA-ergic and cholinergic interneuronal circuits in $\mathrm{M} 1_{\mathrm{HAND}}$, that control the magnitude of SAI (Di Lazzaro et al., 2000; V Di Lazzaro et al., 2005), is comparable between different motor representations (V Di Lazzaro et al., 2005; Vincenzo Di Lazzaro et al., 2005). Interestingly, the individual amount of heterotopic SAF in the FDI muscle scaled positively with the magnitude of homotopic SAI in the ADM muscle. The more homotopic SAI was evoked by electrical stimulation of the little finger, the higher the amount of SAF. This relationship between the magnitude of homotopic SAI and heterotopic SAF was not present, when stimulating the index finger. We speculate that this discrepancy might be related to the more prominent role of the index finger (i.e., the FDI muscle) in the control of dexterous manipulative movements. Therefore, motor representations in $\mathrm{M}_{\mathrm{HAND}}$ controlling SAF of the index finger might be more finely geared to heterotopic sensory input and its resulting homotopic inhibitory effect than motor representations in M1 $1_{\mathrm{HAND}}$ controlling SAF of the little finger.

\subsection{Context dependency of center-surround sensorimotor integration}

Our linear mapping approach enabled us to test the effect of selective contraction of a single target muscle on homotopic SAI and heterotopic SAF without the confounding effect of ongoing EMG activity. Both, muscle-specific facilitatory (heterotopic stimulation) and inhibitory (homotopic stimulation) effects, vanished in the relaxed "surrounding" muscle, when the other intrinsic hand muscle was selectively contracted. This finding indicates that fast cortical sensory-to-motor 
interactions are blocked in relaxed surrounding muscle during tonic contraction of a single hand muscle. This finding confirms previous single-site TMS studies which show a dynamically modification of SAI during movement preparation and execution (Asmussen et al., 2014, 2013; Cho et al., 2016; Classen et al., 2000). The context-dependent loss of homotopic SAI and heterotopic SAF in the surrounding muscle during an isolated tonic contraction may have a stabilizing function for feedforward control of tonic contraction, rendering the $M 1_{\text {HAND }}$ less sensitive to transient sensory input from the hand.

In this context, it is worth mentioning that multiple intracortical mechanisms contribute to fast sensorimotor interactions in human $\mathrm{M} 1_{\mathrm{HAND}}$. Classen et al. (Classen et al., 2000) measured the effect of electrical digit stimulation on the duration of the cortically evoked silent period (CSP) in isometrically contracting intrinsic hand muscles. The duration of the CSP reflects the excitability of intracortical inhibitory circuits that differ from the circuits subserving SAI (Reis et al., 2008). When cutaneous electric stimulation was given $10-50$ ms prior to TMS of the contralateral M1 $1_{\mathrm{HAND}}$, the duration of the cortical silent period became shorter, while the amplitude of the MEP was unaffected (Classen et al., 2000). At the early ISI of $20 \mathrm{~ms}$, the effect of electrical digit stimulation on CSP duration showed a somatotopic gradient with a less pronounced shortening of CSP after heterotopic stimulation (Classen et al., 2000). Together with the present results, these findings show that short-latency cortical sensorimotor integration in $\mathrm{M} 1_{\mathrm{HAND}}$ is dominated by homotopic disinhibition of motor representations during isometric tonic contraction of a single intrinsic hand muscle.

Even at rest, somatotopic organization of sensorimotor representations is subjected to rapid dynamic changes. Our results show that cutaneous electrical digit stimulation induces rapid centreinhibition surround-facilitation in contralateral $\mathrm{M} 1_{\mathrm{HAND}}$, when cutaneous stimulation preceded TMS by 23 ms. However, the single-site TMS study by (Classen et al., 2000) suggests that this pattern may be reversed at longer ISIs. In that study, homotopic electrical stimulation produced centrefacilitation and heterotopic electrical stimulation evoked surround-facilitation at ISIs ranging 
between 150 and $200 \mathrm{~ms}$ (Classen et al., 2000). The dynamic re-arrangement of late sensorimotor interactions, its somatotopical expression and functional significance remain to be addressed with shape-based TMS mapping in future studies.

\section{Conclusion and Outlook}

Using sulcus-shape based mapping, we provide first-time evidence that fast sensorimotor integration in human $\mathrm{M}_{\mathrm{HAND}}$ displays a centre-surround organization, engaging centre inhibition and surrounding facilitation. This sensorimotor centre-surround organization is spatially specific and state dependent. From a biological point of view, this center-specific inhibition and surround facilitation may be an important mechanism to amplify the contrast between the center and the periphery of the sensorimotor receptive field. This mechanism might mediate a rapid and spatially optimized adjustment of cortical excitability in response to transient sensory input from the finger, which might be critical to the acquisition of sensorimotor manual skills and dexterity. Importantly, this center-surround organization and its context-dependent modulation may be disrupted in neurological diseases, such as focal hand dystonia where the precise spatial processing of sensory and motor stimuli is distorted (Tamburin et al., 2002). Here, sulcus-shape based mapping of sensorimotor integration may offer a valuable tool with which to study beneficial and maladaptive sensorimotor reorganization of cortical sensorimotor representations in human $\mathrm{M} 1_{\mathrm{HAND}}$.

\section{Acknowledgments}

This work is part of the project "Biophysically adjusted state-informed cortex stimulation" (BASICS) funded by a synergy grant from Novo Nordisk Foundation (Interdisciplinary Synergy Program 2014; grant number NNF14OC0011413). H.R.S. received additional financial support from the Lundbeck Foundation (Grant of Excellence "Mapping, Modulation and Modelling the Control of Actions"; grant number R59-A5399). 


\section{References}

Asmussen, M.J., Jakobs, M., Lee, K., Zapallow, C.M., Nelson, A.J., 2013. Short-Latency Afferent Inhibition Modulation during Finger Movement. PLoS One 8, e60496.

Asmussen, M.J., Zapallow, C.M., Jacobs, M.F., Lee, K., Tsang, P., Nelson, A.J., 2014. Modulation of short-latency afferent inhibition depends on digit and task-relevance. PLoS One 9, e104807.

Awiszus, F., 2003. Chapter 2 TMS and threshold hunting, Supplements to Clinical Neurophysiology. Elsevier B.V. doi:10.1016/S1567-424X(09)70205-3

Balzamo, E., Marquis, P., Chauvel, P., Régis, J., 2004. Short-latency components of evoked potentials to median nerve stimulation recorded by intracerebral electrodes in the human preand postcentral areas. Clin. Neurophysiol. 115, 1616-1623. doi:10.1016/j.clinph.2004.02.012

Barbas, H., Garcìa-Cabezas, M.A., 2015. Motor cortex layer 4: less is more. Trends Neurosci. 38, 259-261.

Beck, S., Hallett, M., 2011. Surround inhibition in human motor system. Exp. Brain Res. 210, 165172. doi:10.1007/s00221-004-1909-y

Bopp, R., Holler-Rickauer, S., Martin, K.A.C., Schuhknecht, G.F.P., 2017. An Ultrastructural Study of the Thalamic Input to Layer 4 of Primary Motor and Primary Somatosensory Cortex in the Mouse. J Neurosci. 37, 2435-2448.

Catani, M., Dell’Acqua, F., Vergani, F., Malik, F., Hodge, H., Roy, P., Valabregue, R., Thiebaut de Schotten, M., 2012. Short frontal lobe connections of the human brain. Cortex 48, 273-291. doi:10.1016/j.cortex.2011.12.001

Cho, H.J., Panyakaew, P., Thirugnanasambandam, N., Wu, T., Hallett, M., 2016. Dynamic modulation of corticospinal excitability and short-latency afferent inhibition during onset and maintenance phase of selective finger movement. Clin. Neurophysiol. 127, 2343-2349. doi:10.1016/j.clinph.2016.02.020

Churchland, M.M., Shenoy, K. V, 2007. Temporal complexity and heterogeneity of single-neuron activity in premotor and motor cortex. J Neurophysiol 97, 4235-4257. doi:00095.2007 
[pii]ไr10.1152/jn.00095.2007

Classen, J., Steinfelder, B., Liepert, J., Stefan, K., Celnik, P., Cohen, L.G., Hess, a, Kunesch, E., Chen, R., Benecke, R., Hallett, M., 2000. Cutaneomotor integration in humans is somatotopically organized at various levels of the nervous system and is task dependent. Exp. brain Res. 130, 48-59. doi:10.1007/s002210050005

Desmurget, M., Sirigu, A., 2015. Revealing humans' sensorimotor functions with electrical cortical stimulation. Philos. Trans. R. Soc. Lond. B. Biol. Sci. 370. doi:10.1098/rstb.2014.0207

Di Lazzaro, V., Oliviero, A., Pilato, F., Saturno, E., Dileone, M., Marra, C., Daniele, A., Ghirlanda, S., Gainotti, G., Tonali, P.A., 2004. Motor cortex hyperexcitability to transcranial magnetic stimulation in Alzheimer's disease. J. Neurol. Neurosurg. Psychiatry 75, 555-9. doi:10.1136/jnnp.2003.018127

Di Lazzaro, V., Oliviero, A., Profice, P., Pennisi, M.A., Di Giovanni, S., Zito, G., Tonali, P., Rothwell, J.C., 2000. Muscarinic receptor blockade has differential effects on the excitability of intracortical circuits in the human motor cortex. Exp. Brain Res. 135, 455-461. doi:10.1007/s002210000543

Di Lazzaro, V., Oliviero, A., Saturno, E., Dileone, M., Pilato, F., Nardone, R., Ranieri, F., Musumeci, G., Fiorilla, T., Tonali, P., 2005. Effects of lorazepam on short latency afferent inhibition and short latency intracortical inhibition in humans. J. Physiol. 564, 661-8. doi:10.1113/jphysiol.2004.061747

Di Lazzaro, V., Pilato, F., Dileone, M., Tonali, P.A., Ziemann, U., 2005. Dissociated effects of diazepam and lorazepam on short-latency afferent inhibition. J. Physiol. 569, 315-323. doi:10.1113/jphysiol.2005.092155

Di Lazzaro, V., Ziemann, U., 2013. The contribution of transcranial magnetic stimulation in the functional evaluation of microcircuits in human motor cortex. Front. Neural Circuits 7, 18. doi:10.3389/fncir.2013.00018

Dubbioso, R., Pellegrino, G., Antenora, A., De Michele, G., Filla, A., Santoro, L., Manganelli, F., 
2015. The effect of cerebellar degeneration on human sensori-motor plasticity. Brain Stimul. 8, 1144-1150. doi:10.1016/j.brs.2015.05.012

Fabri, M., Burton, H., 1991. Ipsilateral cortical connections of primary somatic sensory cortex in rats. J. Comp. Neurol. 311, 405-424. doi:10.1002/cne.903110310

Ferezou, I., Haiss, F., Gentet, L.J., Aronoff, R., Weber, B., Petersen, C.C.H., 2007. Spatiotemporal Dynamics of Cortical Sensorimotor Integration in Behaving Mice. Neuron 56, 907-923. doi:10.1016/j.neuron.2007.10.007

Friston, K., Kiebel, S., 2009. Cortical circuits for perceptual inference. Neural Networks 22, 10931104. doi:10.1016/j.neunet.2009.07.023

Friston, K.J., Daunizeau, J., Kiebel, S.J., 2009. Reinforcement learning or active inference? PLoS One 4. doi:10.1371/journal.pone.0006421

Hatsopoulos, N.G., Suminski, A.J., 2011. Sensing with the motor cortex. Neuron. doi:10.1016/j.neuron.2011.10.020

Hatsopoulos, N.G., Xu, Q., Amit, Y., 2007. Encoding of movement fragments in the motor cortex. J. Neurosci. 27, 5105-5114. doi:10.1523/JNEUROSCI.3570-06.2007

Hommel, B., 2009. Action control according to TEC (theory of event coding). Psychol. Res. 73, 512-526. doi:10.1007/s00426-009-0234-2

Izraeli, R., Porter, L.L., 1995. Vibrissal motor cortex in the rat: connections with the barrel field. Exp. Brain Res. 104, 41-54. doi:10.1007/BF00229854

Lang, N., Harms, J., Weyh, T., Lemon, R.N., Paulus, W., Rothwell, J.C., Siebner, H.R., 2006. Stimulus intensity and coil characteristics influence the efficacy of rTMS to suppress cortical excitability. Clin. Neurophysiol. 117, 2292-2301. doi:10.1016/j.clinph.2006.05.030

Manganelli, F., Dubbioso, R., Pisciotta, C., Antenora, A., Nolano, M., De Michele, G., Filla, A., Berardelli, A., Santoro, L., 2013. Somatosensory temporal discrimination threshold is increased in patients with cerebellar atrophy. Cerebellum 12, 456-459. doi:10.1007/s12311012-0435-X 
Matyas, F., Sreenivasan, V., Marbach, F., Wacongne, C., Barsy, B., Mateo, C., Aronoff, R.,

Petersen, C.C.H., Penfield, W., Boldrey, E., Donoghue, J.P., Wise, S.P., Georgopoulos, A.P., Schwartz, A.B., Kettner, R.E., Wessberg, J., Serruya, M.D., Hatsopoulos, N.G., Paninski, L., Fellows, M.R., Donoghue, J.P., Graziano, M.S., Taylor, C.S., Moore, T., Brecht, M., Schneider, M., Sakmann, B., Margrie, T.W., Haiss, F., Schwarz, C., Dombeck, D.A., Graziano, M.S., Tank, D.W., Isomura, Y., Harukuni, R., Takekawa, T., Aizawa, H., Fukai, T., Komiyama, T., Brecht, M., Petersen, C.C.H., Diamond, M.E., Heimendahl, M. von, Knutsen, P.M., Kleinfeld, D., Ahissar, E., Ferezou, I., Seidemann, E., Arieli, A., Grinvald, A., Slovin, H., Histed, M.H., Bonin, V., Reid, R.C., Nagel, G., Boyden, E.S., Zhang, F., Bamberg, E., Nagel, G., Deisseroth, K., Arenkiel, B.R., Ayling, O.G., Harrison, T.C., Boyd, J.D., Goroshkov, A., Murphy, T.H., Aronoff, R., Veinante, P., Desch ̃̃anes, M., Hattox, A.M., Priest, C.A., Keller, A., Herfst, L.J., Brecht, M., Jacquin, M.F., Wiegand, M.R., Renehan, W.E., Pinganaud, G., Bernat, I., Buisseret, P., Buisseret-Delmas, C., Hill, D.N., Bermejo, R., Zeigler, H.P., Kleinfeld, D., Coulter, J.D., Jones, E.G., Rathelot, J.A., Strick, P.L., Welker, W.I., Benjamin, R.M., Miles, R.C., Woolsey, C.N., 2010. Motor control by sensory cortex. Science 330, 1240-3. doi:10.1126/science.1195797

Oldfield, R.C., 1971. The assessment and analysis of handedness: The Edinburgh inventory. Neuropsychologia 9, 97-113, doi:10.1016/0028-3932(71)90067-4

Petersen, C.C.H., 2014. Cortical Control of Whisker Movement. Annu. Rev. Neurosci. 37, 183203. doi:10.1146/annurev-neuro-062012-170344

Raffin, E., Pellegrino, G., Di Lazzaro, V., Thielscher, A., Siebner, H.R., 2015. Bringing transcranial mapping into shape: Sulcus-aligned mapping captures motor somatotopy in human primary motor hand area. Neuroimage 120, 164-175. doi:10.1016/j.neuroimage.2015.07.024

Reis, J., Swayne, O.B., Vandermeeren, Y., Camus, M., Dimyan, M.A., Harris-Love, M., Perez, M.A., Ragert, P., Rothwell, J.C., Cohen, L.G., 2008. Contribution of transcranial magnetic stimulation to the understanding of cortical mechanisms involved in motor control. J. Physiol. 
586, 325-351. doi:10.1113/jphysiol.2007.144824

Rossi, S., Hallett, M., Rossini, P.M., Pascual-Leone, A., Safety of TMS Consensus Group, 2009. Safety, ethical considerations, and application guidelines for the use of transcranial magnetic stimulation in clinical practice and research. Clin. Neurophysiol. 120, 2008-39. doi:10.1016/j.clinph.2009.08.016

Sanes, J.N., Donoghue, J.P., 2000. Plasticity and primary motor cortex. Annu. Rev. Neurosci. 23, 393-415. doi:10.1146/annurev.neuro.23.1.393

Tamburin, S., Manganotti, P., Marzi, C.A., Fiaschi, A., Zanette, G., 2002. Abnormal somatotopic arrangement of sensorimotor interactions in dystonic patients. Brain 125, 2719-2730. doi:10.1093/brain/awf279

Tamburin, S., Manganotti, P., Zanette, G., Fiaschi, A., 2001. Cutaneomotor integration in human hand motor areas: Somatotopic effect and interaction of afferents. Exp. Brain Res. 141, 232241. doi:10.1007/s002210100859

Tinazzi, M., Priori, A., Bertolasi, L., Frasson, E., Mauguière, F., Fiaschi, A., 2000. Abnormal central integration of a dual somatosensory input in dystonia. Evidence for sensory overflow. Brain 123, 42-50. doi:10.1093/brain/123.1.42

Tokimura, H., Di Lazzaro, V., Tokimura, Y., Oliviero, A., Profice, P., Insola, A., Mazzone, P., Tonali, P., Rothwell, J.C., 2000. Short latency inhibition of human hand motor cortex by somatosensory input from the hand. J. Physiol. 523 Pt 2, 503-513. doi:PHY_9995 [pii]

Udupa, K., Ni, Z., Gunraj, C., Chen, R., 2014. Effects of short-latency afferent inhibition on shortinterval intracortical inhibition. J. Neurophysiol. 111, 1350-61. doi:10.1152/jn.00613.2013

Udupa, K., Ni, Z., Gunraj, C., Chen, R., 2009. Interactions between short latency afferent inhibition and long interval intracortical inhibition. Exp. Brain Res. 199, 177-183. doi:10.1007/s00221009-1997-9

Yamawaki, N., Borges, K., Suter, B.A., Harris, K.D., Shepherd, G.M.G., 2014. A genuine layer 4 in motor cortex with prototypical synaptic circuit connectivity. Elife 3, e05422. 
doi:10.7554/eLife.05422

Yousry, T.A., Schmid, U.D., Alkadhi, H., Schmidt, D., Peraud, A., Buettner, A., Winkler, P., 1997. Localization of the motor hand area to a knob on the precentral gyrus. A new landmark. Brain 120, 141-157. doi:10.1093/brain/120.1.141

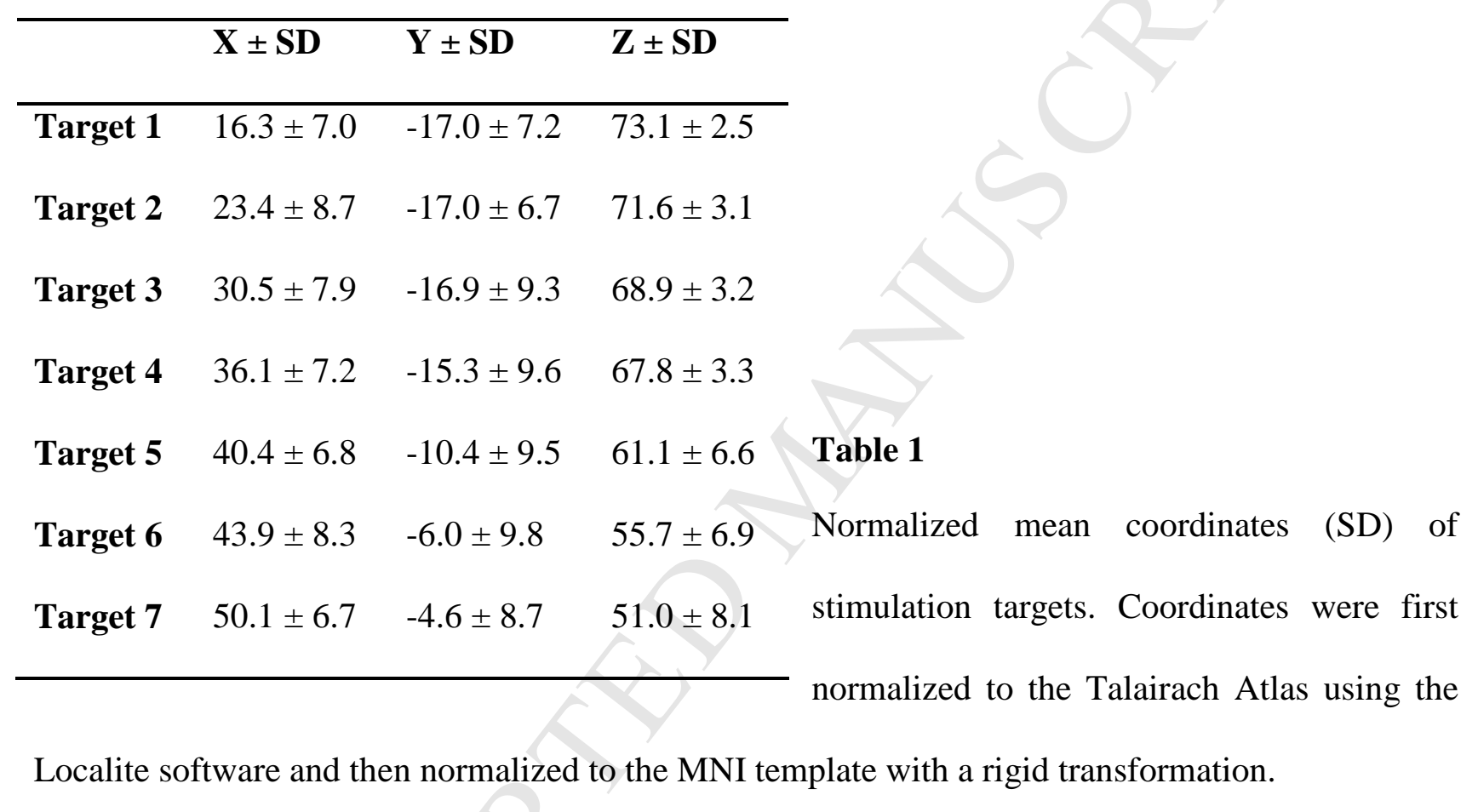




\section{Figure legend}

\section{Figure 1.}

Panel A. Schematic illustration of sulcus-shape based mapping of short-latency afferent sensorimotor interactions. Using frameless stereotaxy, we applied single-pulse transcranial magnetic stimulation (TMS) to one of seven cortical target sites (yellow numbers) in right $\mathrm{M} 1_{\mathrm{HAND}}$ and recorded motor evoked potentials (MEPs) from left first dorsal interosseous (FDI) and abductor digiti minimi (ADM) muscles. Cortical targets 3, 4 and 5 are located in the centre of the hand knob, the macro-anatomical landmark of the $\mathrm{M} 1_{\mathrm{HAND}}$. The blue medial area illustrates the core cortical representation of the $\mathrm{ADM}$ muscle, while the purple lateral area corresponds to the core cortical presentation of the FDI muscle. Note that these muscle representations are overlapping (violet area). Black arrows indicate the coil position or main current direction of the induced electric field perpendicular to the wall of the right central sulcus. The calibration bar equals $10 \mathrm{~mm}$. 
Panel B. Peripheral nerve stimulation was given $23 \mathrm{~ms}$ before a suprathreshold TMS pulse applied to $\mathrm{M} 1_{\mathrm{HAND}}$. The conditioning effects of peripheral stimulation on the motor evoked potentials (MEPs) elicited by the suprathreshold TMS pulse were assessed at each cortical stimulation site to probe short-latency sensorimotor integration in $\mathrm{M} 1_{\mathrm{HAND}}$. Two different types of peripheral stimulation were applied. Homotopic stimulation (labelled as red flash symbol) and heterotopic stimulation (labelled as green flash symbol). Experiment 1 was performed during rest condition. Experiment 2 was performed during tonic muscle contraction (black arrows) of the second or the fifth finger to evaluate the sensorimotor modulatory mechanisms in the surrounding muscles drawn as filled ovals (FDI: pink and ADM: blue).

\section{Figure 2}

Panel A. Homotopic stimulation (in red): the peripheral nerve stimulation is applied close to the TMS muscle target (filled ovals). Heterotopic stimulation (in green). the peripheral nerve stimulation is applied far from the TMS muscle target (filled ovals). ADM= blue. FDI= pink. Homotopic stimulation $=$ full line; heterotopic stimulation $=$ dotted line.

Panel B. Stability of the spatial dissociation (measured as Weigheted Mean Positions) of the two muscle profiles (ADM and FDI) for the unconditioned MEP across the six experimental blocks (Index and little finger stimulated at 100\%-200\%-300\% of perceptual threshold, PT).

Panel C and D. Ratio between the Area under the curve (AUC) of the conditioned MEP amplitudes $\left(A U C_{C}\right)$ and of the unconditioned MEP amplitudes $\left(\mathrm{AUC}_{\mathrm{U}}\right)$ at three different intensities of peripheral stimulation $(100 \%, 200 \%, 300 \%$ of perceptual threshold, PT) for homotopic (C) and heterotopic stimulation (D) $\left(^{*}\right)$ Indicates the significant modulatory effect of the intensity of the peripheral stimulation and of the finger stimulated (homotopic vs heterotopic stimulation) at 300\% of PT. $\mathrm{MEP}_{\mathrm{U}}=$ unconditioned MEP; Homotopic stimulation= full line; heterotopic stimulation = dotted line. $\mathrm{SAI}=$ short afferent inhibition; $\mathrm{SAF}=$ short afferent facilitation. 


\section{Figure 3}

Panel A and B. Mediolateral dissociation of muscle excitability profiles for the conditioned MEP (bold line) and unconditioned MEP (light line), with the FDI muscle located more laterally respect to the ADM muscle either during the homotopic (A) or heterotopic stimulation (B) at PT of $300 \%$. Homotopic stimulation $=$ full line; heterotopic stimulation $=$ dotted line. $\mathrm{SAI}=$ short afferent inhibition. $\mathrm{SAF}=$ short afferent facilitation.

Panel C and D. Weighted Mean Positions of the two muscles profiles referred to the conditioned MEP. (*) Indicates the significant spatial dissociation during homotopic (C) vs heterotopic stimulation (D). Note the somatotopic arrangement is similar either for the homotopic or heterotopic stimulation.

\section{Figure 4}

Panel A. Homotopic and heterotopic stimulation during tonic muscle contraction (black arrows) of the second or the fifth finger to evaluate the sensorimotor modulatory mechanisms in the surrounding muscles drawn as filled ovals (FDI: pink and ADM: blue).

Panel B. Ratio between the area under the curve (AUC) of the conditioned MEP amplitudes (AUCc) and of the unconditioned MEP amplitudes (AUCu) at rest and during selective tonic muscle contraction of an intrinsic hand muscle (Movement) in the relaxed surrounding muscles $(\mathrm{ADM}$ muscle$=$ blue line; FDI muscle= pink line $) .(*)$ indicates the significant abolishment of the homotopic inhibitory and heterotopic facilitatory effects observed at rest condition. HET= heterotopic; $\mathrm{HOM}=$ homotopic.

Panel C. Cortical representation profiles of the surrounding muscles as revealed by the MEP evoked by homotopic or heterotopic stimulation along the seven cortical target positions in 
M1 HAND. The bold lines indicate unconditioned MEP, while the light lines represent the conditioned MEP.

Panel D. Weighted mean positions of the conditioned MEP for surrounding muscles profiles during tonic muscle contraction. The suppression of the facilitation and inhibition was somatotopically expressed with a significant $(*)$ spatial dissociation between the two muscles.

\section{Figure 5}

Panel A. Correlation between heterotopic FDI facilitation and homotopic ADM inhibition (Pearson correlation, $\mathrm{r}=-0.791$, corrected $\mathrm{p}=0.005)$.

Panel B. Correlation between homotopic FDI inhibition and homotopic ADM inhibition (Pearson correlation, $r=0.803$, corrected $\mathrm{p}=0.003)$. 


\section{Table 1}

Normalized mean coordinates (SD) of stimulation targets. Coordinates were first normalized to the

Talairach Atlas using the Localite software and then normalized to the MNI template with a rigid transformation.

\section{$\mathrm{X} \pm \mathrm{SD} \quad \mathrm{Y} \pm \mathrm{SD} \quad \mathrm{Z} \pm \mathrm{SD}$}

\begin{tabular}{cccc}
\hline Target 1 & $16.3 \pm 7.0$ & $-17.0 \pm 7.2$ & $73.1 \pm 2.5$ \\
Target 2 & $23.4 \pm 8.7$ & $-17.0 \pm 6.7$ & $71.6 \pm 3.1$ \\
Target 3 & $30.5 \pm 7.9$ & $-16.9 \pm 9.3$ & $68.9 \pm 3.2$ \\
Target 4 & $36.1 \pm 7.2$ & $-15.3 \pm 9.6$ & $67.8 \pm 3.3$ \\
Target 5 & $40.4 \pm 6.8$ & $-10.4 \pm 9.5$ & $61.1 \pm 6.6$ \\
Target 6 & $43.9 \pm 8.3$ & $-6.0 \pm 9.8$ & $55.7 \pm 6.9$ \\
Target 7 & $50.1 \pm 6.7$ & $-4.6 \pm 8.7$ & $51.0 \pm 8.1$ \\
\hline
\end{tabular}




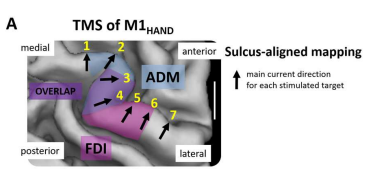

B PERIPHERAL NeRVE StIMULATION

Experiment 1

Experiment 2 


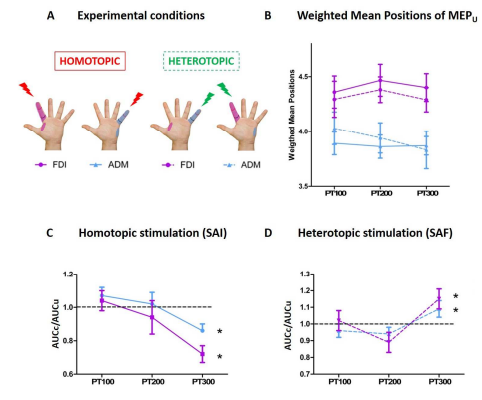




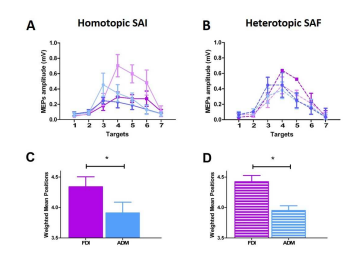




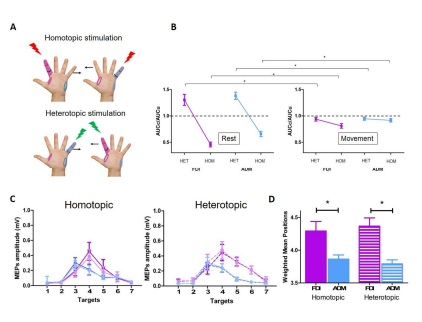


A

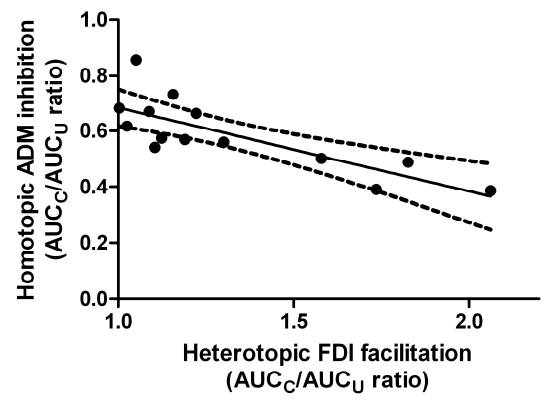

B

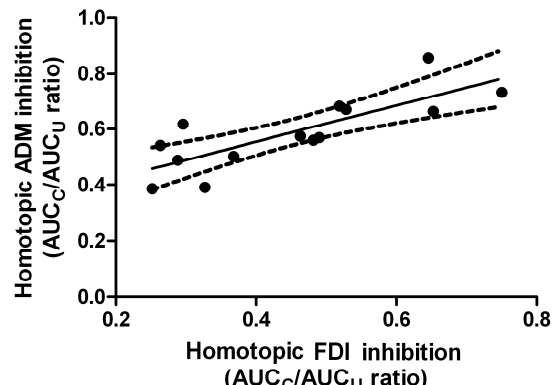

